\title{
Transcutaneous Electrical Nerve Stimulation as a Treatment for Neuropathic Cough: A Tolerability and Feasibility Study
}

\author{
Alexandra Michalowski · Adam Haines · Naum Shaparin · \\ Karina Gritsenko · Alan D. Kaye · Elyse M. Cornett • Michael Z. Lerner (D
}

Received: April 1, 2021 / Accepted: May 1, 2021 / Published online: May 18, 2021

(C) The Author(s) 2021

\begin{abstract}
Transcutaneous electrical nerve stimulation (TENS) is a form of electroanalgesia used for neuropathic pain disorders. Refractory chronic cough, or "neuropathic cough," may be physiologically similar to other neuropathic pain conditions. This study explored the tolerability and feasibility of using TENS as a treatment for neuropathic cough. Laryngeal TENS was administered to five subjects with neuropathic cough. One electrode was placed over the lateral
\end{abstract}

A. Michalowski · A. Haines

Albert Einstein College of Medicine, Bronx, NY, USA

N. Shaparin · K. Gritsenko

Department of Anesthesiology, Albert Einstein

College of Medicine, Bronx, NY, USA

A. D. Kaye · E. M. Cornett

Department of Anesthesiology, LSU Health

Shreveport, Shreveport, LA, USA

M. Z. Lerner $(\bowtie)$

Division of Otolaryngology, Department of Surgery,

Yale School of Medicine, New Haven, CT, USA

e-mail: michael.lerner@yale.edu thyrohyoid membrane, and a second over the cricothyroid space. A frequency of $120 \mathrm{~Hz}$ was applied for $30 \mathrm{~min}$. Participants rated symptoms pre-, during, and post-TENS treatment using a Likert scale. Laryngeal TENS was well tolerated by all subjects. Adverse effects included brief neck discomfort when increasing TENS intensity and an event of mild post-treatment hoarseness. The self-reported scores trended toward a reduction in symptom severity during and after treatment. Controlled trials using this method would elucidate the use of TENS for treatment of patients suffering from chronic cough.

Keywords: Chronic cough; Laryngeal irritability; Neuropathic cough; TENS; Transcutaneous electrical nerve stimulation 


\section{Key Summary Points}

Transcutaneous electrical nerve stimulation (TENS) is a form of electroanalgesia used for neuropathic pain disorders. Refractory chronic cough, or "neuropathic cough," may be physiologically similar to other neuropathic pain conditions.

This study explored the tolerability and feasibility of using TENS as a treatment for neuropathic cough.

Laryngeal TENS was well tolerated by all subjects. Adverse effects included brief neck discomfort when increasing TENS intensity and an event of mild posttreatment hoarseness.

The self-reported scores trended toward a reduction in symptom severity during and after treatment. Controlled trials using this method would elucidate the use of TENS for treatment of patients suffering from chronic cough.

\section{DIGITAL FEATURES}

This article is published with digital features, including a summary slide, to facilitate understanding of the article. To view digital features for this article go to https://doi.org/10.6084/ m9.figshare.14518956.

\section{INTRODUCTION}

Chronic cough, defined as greater than 8 weeks of persistent coughing, is treated using an algorithmic approach that considers common potential etiologies such as laryngopharyngeal reflux, as well as sinonasal, allergic, and pulmonary disease [1]. However, after exhausting the treatment options for these conditions or ruling them out, as many as $12-42 \%$ of patients experience refractory symptoms [2, 3]. It has been theorized that in both refractory chronic cough and neuropathic pain syndromes, altered neural activity due to damage or disease of the peripheral sensory nervous system or its central projections results in states of hypertussia and hyperalgesia, respectively [4-6]. Thus, neuromodulatory medications have been employed to treat refractory chronic cough, or "neuropathic cough" [7, 8]. Oral neuromodulatory medications such as certain antiepileptic drugs (AEDs) and tricyclic antidepressants (TCAs) have provided some therapeutic benefit, but side effects (particularly drowsiness) often limit their utility in a patient population that may be subject to polypharmacy.

Over the last decade, mounting research has supported the notion of refractory chronic cough as a neuropathic condition of the larynx with many similarities to neuropathic pain syndromes. There has been positive literature associating the effects of vagal nerve stimulation with epilepsy, depression, asthma, and the treatment of reactive airway disease. Electrical stimulation of the vagus nerve has been shown to influence the autonomic nervous system as measured by plasma catecholamine levels [9-11].

Transcutaneous electrical nerve stimulation (TENS) is a safe therapeutic option for neuropathic pain [12]. In healthy participants, TENS has been found to raise the somatosensory threshold throughout the stimulated receptive field of the applied current [13]. However, it may have a more marked effect on hypersensitive neurons than on those that are normal [14]. This effect on neuronal hypersensitivity is theorized to underlie the efficacy of TENS in treating neuropathic conditions. Advantages of this noninvasive treatment include its paucity of side effects or drug interactions, its availability, its affordability, and its ease of use [15].

TENS unit settings are an important component of the efficacy of TENS as a therapeutic option for neuropathic conditions. High-frequency and low-frequency TENS therapy have improved pain levels in spinal cord injury patients $[16,17]$. Another factor that has been implicated in the efficacy of TENS for the treatment of neuropathic pain has been the intensity used during TENS therapy [18]. It is 
possible that utilizing the maximum intensity comfortable to the patient may improve the efficacy of TENS therapy. Although studies have suggested specific instrument parameters that may be useful in the treatment of neuropathic pain, there are no studies of whether the device settings that are effective for the treatment of neuropathic pain can translate specifically to the treatment of chronic cough.

There is minimal literature assessing whether a peripheral stimulation device such as a TENS unit could disrupt the neuropathic pain pathway via the vagus nerve along the recurrent laryngeal nerve pathway to mitigate discomfort associated with chronic cough. Prior to assessing the role of TENS as a potential therapeutic option in patients with neuropathic cough, it is first important to determine the range of settings across which laryngeal TENS is both safe and well-tolerated by patients with this condition. We assessed the tolerability and feasibility of laryngeal TENS as a treatment option for patients suffering from neuropathic cough.

\section{METHODS}

This study was completed in accordance with the Albert Einstein College of Medicine Institutional Review Board approval (identification number 2017-7703). The study protocol was designed and implemented in accordance with the Helsinki Declaration of 1964 and all of its later amendments. Informed consent was obtained from all potential participants prior to their decision to participate in this research. This consent process included a section for participants to agree to the use of photography that does not include the participant's face in possible publications that result from this study. All photos obtained and published from this study were obtained and published with consent from the participant. Five participants $(n=5)$ with refractory chronic cough were enrolled in this study. Those with abnormal chest radiograph findings, implanted devices, pregnancy, skin sensory impairment, seizure disorder, or adhesive allergy were excluded from the study. Participants completed the Cough Severity Index (CSI) and Newcastle Laryngeal
Hypersensitivity Questionnaire (NLHQ) to assess the baseline characteristics of their cough $[19,20]$.

Participants were seated in a semi-reclining position. The cricoid cartilage, thyroid cartilage, hyoid bone, and thyrohyoid membrane were identified by palpation and delineated with a surgical skin marker. The TENS LGMedSupply LG-3000 unit was used and its lead wires were attached to $2 \mathrm{~cm} \times 2 \mathrm{~cm}$ self-adhesive electrode pads. For all patients, the cathode was placed over the left thyrohyoid membrane, approximating the location of internal branch of the superior laryngeal nerve, and the anode was placed over the left paramedian cricothyroid space (Fig. 1). Leads were applied unilaterally to the left side to avoid possible bradycardia through stimulation of the right vagus nerve [21]. Prior to electrode placement, the left neck skin was cleansed with an alcohol pad and electrodes were placed by the principal investigator and secured with tape.

To assess tolerability, flexible laryngoscopy was performed without the use of intranasal lidocaine or decongestant so as to eliminate the potential confounding effect of topical anesthesia. The flexible laryngoscope was introduced prior to TENS activation. As the TENS unit was powered on and gradually increased to the maximum tolerated intensity, the larynx was monitored for any abnormal motion, spasm, or muscle contraction deemed outside of the normal range of motion on a standard laryngologist assessment. After the maximum tolerated intensity was reached, the flexible laryngoscope was withdrawn, and the patient underwent continued TENS treatment. Visual inspection of the external anterior cervical musculature was also observed for contractions. To assess feasibility, visual inspection was also used to assess pad movement. Patients were asked to report if stimulation was occurring over the desired area.

Treatment consisted of two 15-min sessions with a 2-min break in between. During each of the sessions, the unit was kept at a high frequency $(120 \mathrm{~Hz})$ and a short pulse duration $(120 \mu \mathrm{s})$, in keeping with the conventional TENS model of neuropathic pain treatment [22]. Intensity was titrated to the maximum tolerated 


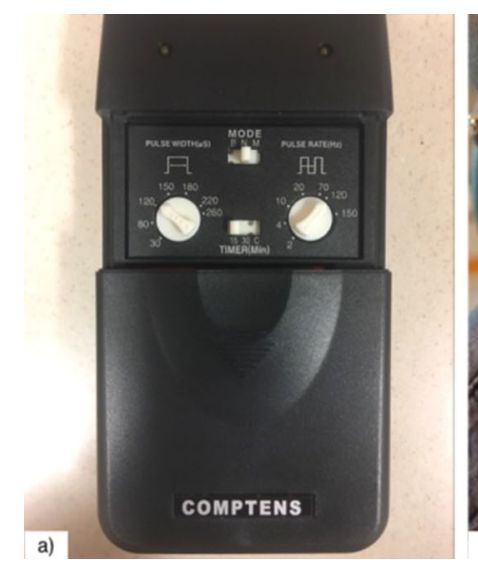

Fig. 1 Transcutaneous electrical nerve stimulation (TENS) application method. a The LGMedSupply LG3000 unit was utilized to provide TENS treatment to each participant. The adjustable settings for this device include pulse width, mode, pulse rate, amplitude of stimulation, as well as a timer that can be set for $15 \mathrm{~min}, 30 \mathrm{~min}$, or continuous stimulation. $\mathbf{b}$ This photograph of the anterior neck demonstrates the placement of TENS electrode leads and outlines important anatomical landmarks. The

intensity and maintained at these settings for the duration of each session. The subjects rated symptoms pre-, during, and post-TENS treatment using a 5-point Likert scale. Each participant was asked, "On a scale of 0 to 4 , with 4 being the worst your symptoms have been, could you rate the severity of the symptoms pertaining to your cough?"

\section{RESULTS}

All patients were female and the mean age was 73 years. Two of the participants were black, one was white, and two were Hispanic or Latin American. The mean CSI score was 25.6 and the mean NLHQ score was 14.8 (Table 1 ).

Tolerability outcomes were met for all participants. No participants were able to tolerate an intensity greater than $3 \mathrm{~mA}$. As such, all participants were maintained at an intensity of $3 \mathrm{~mA}$ for the duration of each session. Adverse effects were limited to mild neck discomfort for up to several seconds after increasing the TENS intensity, which resolved when the intensity was lowered to $3 \mathrm{~mA}$. Neck discomfort did not cathode, indicated by the red electrode, was placed over the left thyrohyoid membrane, and the anode, indicated by the black electrode, was placed over the left paramedian cricothyroid space. c This photograph demonstrates the method of surgical tape placement over the TENS electrode pads. This was kept in place throughout the TENS treatment in order to maintain complete skin adherence

persist following the TENS therapy for any of the participants. New-onset mild hoarseness was noticed and reported by one patient at the conclusion of the treatment. The exact duration of hoarseness was not recorded, but it had completely resolved by $24 \mathrm{~h}$. Normal vocal cord motion was observed, and there were no cases of laryngospasm or involuntary vocal fold adduction or contraction. During the study, the pads were visualized to maintain appropriate positioning without movement, and the patients reported stimulation in the appropriate areas.

Comparison of Likert scores demonstrated an overall decrease in irritability (Table 2). The change in symptom severity rating decreased by an average of 0.8 points from before to during electrical stimulation, and the average symptom severity rating decreased by 0.2 points during treatment to $1 \mathrm{~min}$ after treatment completion. Three of the five patients reported symptom improvement during and immediately after TENS treatment. 
Table 1 Cough severity survey scores: this table displays pretreatment subject scores for both the Newcastle Laryngeal Hypersensitivity Questionnaire and the Cough Severity Index

\begin{tabular}{lrrrrrr}
\hline Baseline symptom surveys & \multicolumn{9}{c}{ Participant number } \\
\cline { 2 - 8 } & $\mathbf{0 0 1}$ & $\mathbf{0 0 2}$ & $\mathbf{0 0 3}$ & $\mathbf{0 0 4}$ & $\mathbf{0 0 5}$ & Total survey means \\
\hline Newcastle total obstruction score & 36.0 & 30.0 & 36.0 & 20.0 & 43.0 & \\
Newcastle total pain/thermal score & 20.0 & 18.0 & 16.9 & 13.0 & 13.0 & \\
Newcastle total throat tickle score & 20.0 & 9.0 & 13.0 & 20.0 & 19.0 & \\
Newcastle average obstruction score & 4.5 & 3.8 & 4.5 & 2.5 & 5.4 & \\
Newcastle average pain/thermal score & 6.7 & 6.0 & 5.3 & 4.3 & 4.3 & \\
Newcastle average throat tickle score & 6.7 & 3.0 & 4.3 & 6.7 & 6.3 & \\
Total Newcastle LHQ (sum of average scores) & 18.0 & 13.0 & 14.0 & 13.0 & 16.0 & $\mathbf{1 4 . 8}$ \\
Total CSI & 30.0 & 9.0 & 27.0 & 33.0 & 29.0 & $\mathbf{2 5 . 6}$
\end{tabular}

The NLHQ categorized its questions into assessments of three major qualities of laryngeal irritability: obstruction, thermal sensation/pain, and throat tickling sensation. The CSI served as a baseline for the severity of perceived symptoms for each subject

Table 2 Likert scale assessment of cough symptoms: this table shows patients' perceptions of their cough symptom severity before beginning the TENS intervention, during their mid-treatment 2-min break, and immediately following discontinuation of the treatment for each subject

\begin{tabular}{|c|c|c|c|c|c|c|}
\hline \multirow{2}{*}{$\begin{array}{l}\text { Likert } \\
\text { scale } \\
\text { scores }\end{array}$} & \multicolumn{5}{|c|}{ Participant number } & \multirow[b]{2}{*}{$\begin{array}{l}\text { Likert time } \\
\text { point mean }\end{array}$} \\
\hline & $\overline{001}$ & 002 & 003 & 004 & 005 & \\
\hline Before & 0.0 & 3.0 & 2.0 & 1.0 & 4.0 & 2.0 \\
\hline During & 0.0 & 1.0 & 0.0 & 2.0 & 3.0 & 1.2 \\
\hline After & 0.0 & 1.0 & 0.0 & 2.0 & 2.0 & 1.0 \\
\hline
\end{tabular}

The mean of each time point is included in the table

\section{DISCUSSION}

A single case report from 1987 describes subjective success at aborting a patient's 9-year cough using TENS. That report lacks details, including application technique, TENS settings, duration of treatment, and rationale for use in that particular context [23]. Our study describes an anatomically based application technique and a proposed treatment paradigm for the use of laryngeal TENS. The modality utilized highfrequency and low-intensity settings to stimulate large diameter A- $\beta$ afferent fibers, which inhibit nociceptor activity, thereby reducing hyperalgesia [9]. Higher intensities have also been implicated in the efficacy of TENS for the treatment of neuropathic pain, suggesting that utilizing the maximum tolerable intensity may improve therapeutic success [18].

We demonstrated that TENS use for a chronic cough is tolerable, feasible, and showed a trend toward improved symptoms during and immediately after treatment. Limitations include a small and homogeneous sample, which may have resulted in nongeneralizable false-positive findings. Further research with larger sample sizes would be necessary prior to making concrete statements regarding therapeutic efficacy. Future studies that are designed to evaluate the long-term benefits of TENS application in a controlled office setting would be beneficial. Additionally, since there is currently no evidence substantiating TENS as a standalone treatment for neuropathic cough, it would also be beneficial to study TENS administration as an adjunct treatment. 


\section{CONCLUSIONS}

Participants tolerated TENS treatment and experienced a subjective improvement in symptoms and minimal adverse effects during and immediately after treatment. Patient-reported stimulation of the appropriate anatomic area that corresponded to their symptoms demonstrated feasibility. This study can inform future research by providing evidence of tolerable TENS device settings and methods for application.

\section{ACKNOWLEDGEMENTS}

We would like to thank all of the participants in this study for their contribution to the advancement of medical knowledge. The work was performed at Albert Einstein College of Medicine, Bronx, New York, USA.

Funding. No funding or sponsorship was received for this study or publication of this article.

Authorship. All named authors meet the International Committee of Medical Journal Editors (ICMJE) criteria for authorship for this article, take responsibility for the integrity of the work as a whole, and have given their approval for this version to be published.

Authors' Contributions. AM, NS, MZL contributed to the concept and design of the study. $\mathrm{AM}, \mathrm{AH}$, and MZL implemented the study protocol. AM, AH, NS, KG, ADK, MZL, and EMC all contributed to interpretation of results and preparation of the written manuscript. All authors agreed to the final version of the written manuscript and agree to be accountable for all aspects of the work. The principal investigator ML had full access to all the data in the study and takes full responsibility for the integrity of the data and the accuracy of the data analysis.

Prior Publication. This manuscript is based on work that was also presented as a poster presentation at the American Laryngological Association's 2018 Annual Meeting at COSM in National Harbor, Maryland on April 20, 2018.

Disclosures. Alexandra Michalowski, Adam Haines, Naum Shaparin, Karina Gritsenko, Alan David Kaye, Michael Z Lerner, and Elyse M. Cornett have no financial relationships or conflicts of interest to disclose. Alexandra Michalowski has since changed affiliations and is currently affiliated with New York Presbyterian Hospital, New York, New York.

Compliance with Ethics Guidelines. This study was completed in accordance with the Einstein Institutional Review Board approval (identification number 2017-7703). The study protocol was designed and implemented in accordance with the Helsinki Declaration of 1964 and all of its later amendments. Informed consent was obtained from all potential participants prior to their decision to participate in this research. This consent process included a section for participants to agree to the use of photography that does not include the participants face for use in possible publications that result from this study. All photos obtained and published from this study were obtained and published with consent from the participant.

Data Availability. The datasets generated during and/or analyzed during the current study are available from the corresponding author on reasonable request.

Open Access. This article is licensed under a Creative Commons Attribution-NonCommercial 4.0 International License, which permits any non-commercial use, sharing, adaptation, distribution and reproduction in any medium or format, as long as you give appropriate credit to the original author(s) and the source, provide a link to the Creative Commons licence, and indicate if changes were made. The images or other third party material in this article are included in the article's Creative Commons licence, unless indicated otherwise in a credit line to the material. If material is not included in the article's Creative Commons licence and your intended use is not permitted by statutory 
regulation or exceeds the permitted use, you will need to obtain permission directly from the copyright holder. To view a copy of this licence, visit http://creativecommons.org/licenses/by$\mathrm{nc} / 4.0 /$.

\section{REFERENCES}

1. Pratter $\mathrm{MR}$, et al. An algorithmic approach to chronic cough. Ann Intern Med. 1993;119(10): 977-83.

2. Haque RA, Usmani OS, Barnes PJ. Chronic idiopathic cough: a discrete clinical entity? Chest. 2005;127(5):1710-3.

3. Kardos P. Proposals for a rationale and for rational diagnosis of coughs. Pneumologie. 2000;54(3): $110-5$.

4. Mazzone SB, et al. Investigation of the neural control of cough and cough suppression in humans using functional brain imaging. J Neurosci. 2011;31(8):2948-58.

5. Farrell MJ, Mazzone SB. Sensations and regional brain responses evoked by tussive stimulation of the airways. Respir Physiol Neurobiol. 2014;204: 58-63.

6. Niimi A, Chung KF. Evidence for neuropathic processes in chronic cough. Pulm Pharmacol Ther. 2015;35:100-4.

7. Ryan NM, Birring SS, Gibson PG. Gabapentin for refractory chronic cough: a randomised, doubleblind, placebo-controlled trial. Lancet. 2012;380(9853):1583-9.

8. Cohen SM, Misono S. Use of specific neuromodulators in the treatment of chronic, idiopathic cough: a systematic review. Otolaryngol Head Neck Surg. 2013;148(3):374-82.

9. Hoffmann TJ, et al. Inhibition of histamine-induced bronchoconstriction in Guinea pig and Swine by pulsed electrical vagus nerve stimulation. Neuromodulation. 2009;12(4):261-9.

10. Hoffmann TJ, et al. Low voltage vagal nerve stimulation reduces bronchoconstriction in guinea pigs through catecholamine release. Neuromodulation. 2012;15(6):527-36.
11. Hatton KW, et al. Vagal nerve stimulation: overview and implications for anesthesiologists. Anesth Analg. 2006;103(5):1241-9.

12. Vance CG, et al. Using TENS for pain control: the state of the evidence. Pain Manag. 2014;4(3): 197-209.

13. Dean J, Bowsher D, Johnson MI. The effects of unilateral transcutaneous electrical nerve stimulation of the median nerve on bilateral somatosensory thresholds. Clin Physiol Funct Imaging. 2006;26(5):314-8.

14. Cheing GL, Luk ML. Transcutaneous electrical nerve stimulation for neuropathic pain. J Hand Surg Br. 2005;30(1):50-5.

15. Johnson MI, Bjordal JM. Transcutaneous electrical nerve stimulation for the management of painful conditions: focus on neuropathic pain. Expert Rev Neurother. 2011;11(5):735-53.

16. Norrbrink C. Transcutaneous electrical nerve stimulation for treatment of spinal cord injury neuropathic pain. J Rehabil Res Dev. 2009;46(1):85-93.

17. Celik EC, et al. The effect of low-frequency TENS in the treatment of neuropathic pain in patients with spinal cord injury. Spinal Cord. 2013;51(4):334-7.

18. Wang B, et al. Effect of the intensity of transcutaneous acupoint electrical stimulation on the postoperative analgesic requirement. Anesth Analg. 1997;85(2):406-13.

19. Shembel AC, et al. Development and validation of the cough severity index: a severity index for chronic cough related to the upper airway. Laryngoscope. 2013;123(8):1931-6.

20. Vertigan AE, Bone SL, Gibson PG. Development and validation of the Newcastle laryngeal hypersensitivity questionnaire. Cough. 2014;10(1):1.

21. Howland RH. Vagus nerve stimulation. Curr Behav Neurosci Rep. 2014;1(2):64-73.

22. Johnson M. Transcutaneous electrical nerve stimulation: mechanisms. Clin Appl Evid Rev Pain. 2007;1(1):7-11.

23. Rogers C. Nine-year cough aborted by TENS. J Tenn Med Assoc. 1987;80(2):81-2. 